\title{
TOTAL BODY WATER AND TOTAL EXCHANGEABLE SODIUM IN EDEMATOUS STATES DUE TO CARDIAC, RENAL OR HEPATIC DISEASE ${ }^{1}$
}

\author{
By SAUL J. FARBER AND ROBERT J. SOBERMAN \\ (From the Department of Medicine, New York University College of Medicine and the Third \\ (New York University) Medical Division, Bellevue Hospital, New York, N. Y.)
}

(Submitted for publication August 25, 1955 ; accepted March 15, 1956)

The physiological defects of salt and water retention appear to be the same in diseases of various etiologies associated with edema. However, the relative amounts of sodium and water retained and their distribution may be different and related to the metabolic faults of the particular disease. Although the concentrations of electrolytes in extracellular fluid are similar in edematous states, the concentrations in other compartments may indicate differences related to the relative accumulation and distribution of sodium and water.

At present, methods are available to determine the concentrations of electrolytes in extracellular fluid. In intact man, concentrations of ions in other compartments of the body have to be indirectly determined. One such assay may be attempted by computing the body concentration of the electrolyte from the determinations of total exchangeable body content of the electrolyte and total body water. The value so derived does not have the physical chemical significance of concentration but may be indicative of changes occurring in the extracellular fluid as well as in other body compartments.

Employing this procedure, the present study attempts to differentiate various clinical conditions with edema on the basis of total exchangeable sodium and body water content. In particular, patients with congestive heart failure have been compared to patients with other diseases associated with edema. The data indicate that patients with congestive heart failure apparently have a high body concentration of sodium (mEq. of exchangeable sodium per liter of body water). This in-

\footnotetext{
1 Major support for this study came from a grant (H-1270) of the United States Public Health Service, National Heart Institute. Additional support was given by the Life Insurance Medical Research Fund, the Atomic Energy Commission and some of the work was done under the sponsorship of the Commission on Hemorrhagic Fever.
}

crease in the ratio of body sodium to body water persists in the majority of patients with heart failure after they have been rendered free of edema. A difference also exists between edematous patients with heart disease and edematous patients with hepatic or renal disease in body water and body water expressed as a per cent of body weight. The patients with edema due to heart disease have less body water and body water as the per cent of body weight than do edematous patients with liver or renal disease.

\section{SUBJECTS AND METHODS}

\section{Subjects}

The control group studied consisted of 27 patients without edema and with no evidence of renal, liver or heart disease. Among these subjects were 5 patients who had recent marked reduction in weight due to neoplastic disease. These 5 patients were intended to serve as a control group for heart disease patients who may have lost weight before or during the study.

The heart disease group consisted of 50 patients with edema and 33 patients who were previously edematous. Of the latter group of 33 subjects, 13 were studied in the edematous state and restudied when they were free of edema. They were rendered free of edema by bed rest, digitalis and/or mercurial diuretics and remained at a constant weight for at least 3 weeks. The other 20 patients in this non-edematous category were clinically free of edema for a period of several months to one year. The heart disease patients included individuals with hypertensive, arteriosclerotic, rheumatic or unknown types of heart disease.

The hepatic and renal groups studied consisted of 16 subjects with edema and/or ascites due to cirrhosis of the liver and 10 subjects with edema due to renal disease. The patients with renal disease had either acute glomerulo-nephritis or the nephrotic syndrome.

\section{Methods}

Body water. Body water was determined by the antipyrine dilution method (1). After drawing blood for a blank serum determination, $20 \mathrm{ml}$. of 5 per cent antipyrine were injected from a calibrated syringe. In the non- 
TABLE I

Comparison of body water as determined by dilution of antipyrine and deuterium in edematous patients with heart disease

\begin{tabular}{ccc}
\hline \hline Patient & $\begin{array}{c}\text { Antipyrine } \\
\text { space } \\
\text { (Liters) }\end{array}$ & $\begin{array}{c}\text { Deuterium } \\
\text { space } \\
\text { (Liters) }\end{array}$ \\
\hline 1 & 45.3 & 47.2 \\
2 & 49.8 & 51.0 \\
3 & 64.0 & 62.8 \\
4 & 51.5 & 50.0 \\
5 & 33.5 & 35.9 \\
6 & 47.2 & 48.2 \\
7 & 38.2 & 40.8 \\
8 & 50.6 & 50.6 \\
9 & 41.0 & 41.8 \\
Mean & 46.8 & 47.6 \\
\hline
\end{tabular}

edematous patients blood samples were drawn 5, 7 and 9 hours after the injection of antipyrine. In the patients with edema, specimens of blood were drawn $7,9,11,13$, and in some instances 18 and 24 hours after the injection
TABLE II

Concentration of antipyrine in plasma and extravascular fluids

\begin{tabular}{cccc}
\hline & & \multicolumn{2}{c}{ Ratio of $\frac{\text { Fluid concentration }}{\text { Plasma concentration }} \times 100$} \\
\cline { 3 - 4 } $\begin{array}{c}\text { Fluid } \\
\text { source }\end{array}$ & Number & Mean & Range \\
\hline Edema & 16 & 98.4 & $83-111$ \\
Ascitic & 5 & 99.3 & $94-103$ \\
Pleural & 2 & 102.0 &
\end{tabular}

* These values were determined on samples taken 10 to 24 hours after the injection of antipyrine.

of antipyrine. The concentrations of antipyrine were determined by accepted chemical procedures (2).

The validity of the antipyrine method for the determination of body water in normals and patients with abnormal fluid accumulation due to liver disease has been established in other studies $(1,3)$. Since a reduced rate of blood flow may hamper equilibration in body fluids of any injected substance it was necessary to validate the

TABLE III

Exchangeable sodium and body water in patients without clinical evidence of heart, renal or hepatic disease-"controls"

\begin{tabular}{|c|c|c|c|c|c|c|c|}
\hline Patient & $\begin{array}{l}\text { Weight } \\
\text { (Kilos) }\end{array}$ & $\underset{\substack{\text { Serum } \\
\text { sodium } \\
(m E q . / L .)}}{ }$ & $\begin{array}{l}\text { Anti- } \\
\text { pyrine } \\
\text { space } \\
(\text { Liters })\end{array}$ & $\begin{array}{c}\begin{array}{c}\text { Total } \\
\text { exchange- } \\
\text { able } \\
\text { sodium } \\
(m E q .)\end{array}\end{array}$ & $\frac{\frac{\text { A.S.* }}{W t_{\text {t. }}}}{(\% \text { Body wt.) }}$ & $\frac{\text { TENa* }}{(m E \text { A.S. } / L .)}$ & $\frac{T E N a^{*}}{W t}$ \\
\hline $\begin{array}{c}1 \\
2 \\
3 \\
4 \dagger \\
5 \\
6 \\
7 \\
8 \\
9 \dagger \\
10 \\
11 \\
12 \\
13 \\
14 \\
15 \\
16 \\
17 \\
18 \\
19 \\
20 \dagger \\
21 \dagger \\
22 \dagger \\
23 \\
24 \\
25 \\
26 \\
27\end{array}$ & $\begin{array}{r}68.2 \\
60.9 \\
54.1 \\
38.6 \\
69.6 \\
68.6 \\
70.5 \\
81.8 \\
55.0 \\
80.9 \\
98.1 \\
74.6 \\
71.9 \\
79.1 \\
37.0 \\
57.3 \\
101.5 \\
71.5 \\
71.5 \\
59.5 \\
52.4 \\
59.5 \\
80.5 \\
68.7 \\
47.3 \\
55.0 \\
73.2\end{array}$ & $\begin{array}{l}139 \\
144 \\
134 \\
136 \\
136 \\
136 \\
127 \\
144 \\
135 \\
148 \\
132 \\
138 \\
137 \\
142 \\
140 \\
139 \\
141 \\
130 \\
135 \\
142 \\
145 \\
131 \\
141 \\
134 \\
137 \\
141 \\
141\end{array}$ & $\begin{array}{l}35.2 \\
31.1 \\
35.0 \\
27.4 \\
36.5 \\
45.5 \\
34.0 \\
35.0 \\
32.6 \\
40.5 \\
41.1 \\
40.7 \\
38.6 \\
48.6 \\
25.3 \\
26.3 \\
48.0 \\
33.4 \\
35.5 \\
29.1 \\
28.8 \\
33.5 \\
42.5 \\
36.8 \\
26.1 \\
31.5 \\
35.3\end{array}$ & $\begin{array}{l}2,840 \\
2,600 \\
2,550 \\
2,320 \\
3,280 \\
2,920 \\
2,480 \\
3,030 \\
3,050 \\
3,240 \\
3,630 \\
3,310 \\
3,060 \\
3,470 \\
2,130 \\
2,100 \\
3,950 \\
2,700 \\
2,760 \\
2,540 \\
2,590 \\
2,790 \\
3,440 \\
3,160 \\
2,040 \\
2,550 \\
2,080\end{array}$ & $\begin{array}{l}\mathbf{5 1 . 6} \\
51.1 \\
64.6 \\
71.0 \\
52.4 \\
65.0 \\
48.5 \\
42.8 \\
41.5 \\
50.0 \\
41.9 \\
54.6 \\
53.6 \\
61.5 \\
68.4 \\
45.9 \\
45.5 \\
46.8 \\
49.6 \\
49.0 \\
55.0 \\
56.3 \\
52.9 \\
53.6 \\
55.2 \\
57.3 \\
48.2\end{array}$ & $\begin{array}{l}80.8 \\
83.6 \\
73.0 \\
85.0 \\
88.5 \\
64.0 \\
73.0 \\
86.6 \\
93.0 \\
80.0 \\
88.5 \\
81.4 \\
79.2 \\
71.4 \\
84.0 \\
80.0 \\
78.0 \\
80.8 \\
77.8 \\
87.5 \\
90.0 \\
83.3 \\
81.0 \\
86.0 \\
78.2 \\
82.0 \\
75.9\end{array}$ & $\begin{array}{l}41.7 \\
42.7 \\
47.1 \\
60.1 \\
47.1 \\
42.6 \\
35.2 \\
37.1 \\
55.5 \\
40.1 \\
37.0 \\
44.4 \\
42.6 \\
43.9 \\
57.6 \\
36.7 \\
38.9 \\
37.8 \\
38.6 \\
42.7 \\
49.5 \\
46.9 \\
42.7 \\
46.0 \\
43.2 \\
46.4 \\
36.6\end{array}$ \\
\hline \multirow{3}{*}{$\begin{array}{c}\text { Mean } \\
\text { Standard } \\
\text { deviation } \\
\text { Standard } \\
\text { error }\end{array}$} & $\overline{66.8}$ & $\overline{138}$ & $\overline{35.0}$ & $\overline{2,896}$ & $\overline{52.0}$ & 81.3 & 43.7 \\
\hline & 15.3 & 4.9 & 7.0 & 479 & 6.3 & 6.2 & 5.9 \\
\hline & 3.0 & 0.99 & 1.2 & 94 & 1.3 & 1.2 & 1.2 \\
\hline
\end{tabular}

* In all tables: A.S.-Antipyrine Space, TENa-Total Exchangeable Sodium, TENa/AS-Exchangeable Sodium per liter of Antipyrine Space (Body Water), TENa/Kg.-Exchangeable Sodium per kg. Body Weight.

$\dagger$ Patients with recent loss of weight due to neoplastic disease. 
BODY WATER AND EXCHANGEABLE SODIUM IN EDEMATOUS STATES

TABLE IV

Exchangeable sodium and body water in patients with edema due to heart disease

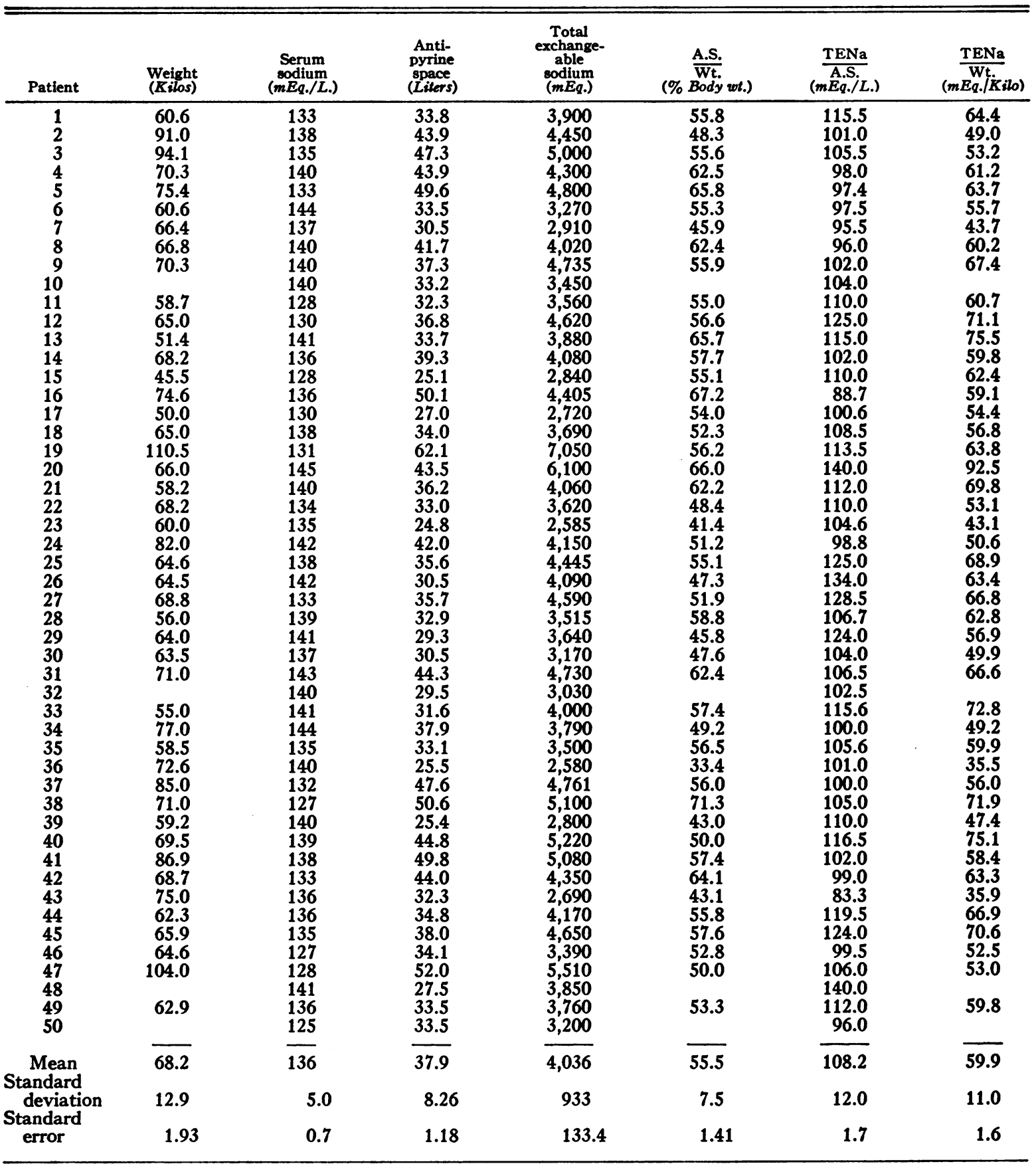

antipyrine dilution method as a measure of body water in patients with edema due to congestive heart failure. Accordingly, simultaneous determinations of total body water by deuterium and antipyrine dilution were performed in nine markedly edematous patients with congestive heart failure. Deuterium was administered intravenously by a pump with a burette as a reservoir. Serum concentration was determined by the mass spectrometer. 2

As a further aid in establishing the antipyrine method as a measure of body water in patients with abnormal fluid accumulations, equilibration of antipyrine between

${ }^{2} \mathrm{We}$ are indebted to Mr. Irving Sucher of the Department of Chemistry, College of Physicians and Surgeons, for these determinations. 
blood and large extravascular collections of fluid was determined. These fluids consisted of 23 samples of edema, ascitic and pleural fluid studied at various intervals after the injection of antipyrine.

The sources of the extravascular fluids studied were as follows: 16 edema fluids: 14 from patients with heart disease, one from a patient with cirrhosis of the liver, and one from a patient with edema of unknown cause; 5 ascitic fluids: one specimen from a patient with heart disease and four from patients with cirrhosis of the liver; 2 pleural fluids: a specimen from a patient with heart disease and one from a patient with renal disease.

Total exchangeable sodium. Total exchangeable sodium was determined by the dilution of radioisotopic sodium (4-6). One ml. of $\mathrm{Na}^{24}$ containing 100 microcuries was injected from a tuberculin syringe. Twenty-four hours were allowed for mixing and equilibration of the injected radioactive element with the exchangeable body sodium pool. Twenty-four and twenty-six hours after the injection samples of blood were drawn and serum analyzed. If these determinations did not agree within
5 per cent, a third and if necessary a fourth sample was drawn at hourly intervals until the concentration remained constant. Specimens of edema, ascitic and pleural fluid drawn twenty-four hours after injection of $\mathrm{Na}^{2}$ were compared for radioactivity with serum drawn at the same time.

In most instances the loss of radiosodium in the urine was not over one per cent of the administered sodium. When this value was above one per cent, it was subtracted from the injected dose.

Serum sodium was determined by the use of an internal standard flame photometer.

\section{RESULTS}

Comparison of body water by antipyrine and deuterium dilution methods in patients with congestive heart failure

The spaces of distribution of antipyrine and deuterium were very similar, antipyrine 46.8 liters,

TABLE V

Exchangeable sodium and body water in patients with heart disease who were previously edematous

\begin{tabular}{|c|c|c|c|c|c|c|c|}
\hline Patient & $\begin{array}{l}\text { Weight } \\
\text { (Kilos) }\end{array}$ & $\underset{\substack{\text { Serum } \\
\text { sodium } \\
(m E q . / L .)}}{\operatorname{cod}}$ & $\begin{array}{l}\text { Anti- } \\
\text { pyrine } \\
\text { space } \\
\text { (Liters) }\end{array}$ & $\begin{array}{c}\text { Total } \\
\text { exchange- } \\
\text { able } \\
\text { sodium } \\
(m E q .)\end{array}$ & 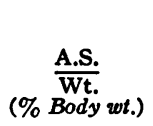 & $\frac{\text { TENa }}{(m E \text { A.S. } / L .)}$ & $\frac{\text { TENa }}{\mathrm{Wt}_{(m E q . / K i l o)}}$ \\
\hline $\begin{array}{r}1 \\
2 \\
3 \\
4 \\
5 \\
6 \\
7 \\
8 \\
9 \\
10 \\
11 \\
12 \\
13 \\
14 \\
15 \\
16 \\
17 \\
18 \\
19 \\
20 \\
21 \\
22 \\
23 \\
24 \\
25 \\
26 \\
27 \\
28 \\
29 \\
30 \\
31 \\
32 \\
33\end{array}$ & $\begin{array}{r}36.4 \\
65.0 \\
66.8 \\
76.4 \\
61.6 \\
77.3 \\
53.6 \\
78.7 \\
66.9 \\
49.1 \\
61.8 \\
66.4 \\
63.7 \\
70.0 \\
61.3 \\
77.8 \\
47.7 \\
56.4 \\
77.3 \\
58.2 \\
63.8 \\
\\
54.5 \\
61.3 \\
74.1 \\
52.3 \\
51.4 \\
69.0 \\
57.7 \\
52.0 \\
109.5 \\
60.0 \\
61.5\end{array}$ & $\begin{array}{l}138 \\
132 \\
140 \\
140 \\
139 \\
140 \\
136 \\
139 \\
140 \\
134 \\
140 \\
141 \\
135 \\
137 \\
137 \\
134 \\
141 \\
141 \\
138 \\
138 \\
140 \\
147 \\
138 \\
141 \\
140 \\
140 \\
135 \\
140 \\
135 \\
139 \\
140 \\
142 \\
138\end{array}$ & $\begin{array}{l}27.4 \\
42.1 \\
37.3 \\
36.2 \\
37.8 \\
37.2 \\
27.0 \\
43.5 \\
29.0 \\
24.1 \\
28.2 \\
30.2 \\
31.8 \\
41.1 \\
43.0 \\
41.3 \\
29.2 \\
30.9 \\
38.5 \\
36.1 \\
32.0 \\
22.6 \\
25.0 \\
30.6 \\
37.2 \\
32.0 \\
32.4 \\
25.0 \\
31.8 \\
27.0 \\
56.2 \\
35.8 \\
38.2\end{array}$ & $\begin{array}{l}2,260 \\
3,370 \\
3,050 \\
3,920 \\
3,060 \\
4,660 \\
2,650 \\
4,395 \\
3,472 \\
2,420 \\
2,800 \\
3,370 \\
2,620 \\
3,540 \\
3,700 \\
3,850 \\
2,670 \\
3,170 \\
3,630 \\
2,940 \\
3,680 \\
2,295 \\
2,630 \\
3,350 \\
3,880 \\
3,360 \\
2,760 \\
2,730 \\
3,595 \\
3,060 \\
5,760 \\
3,950 \\
4,360\end{array}$ & $\begin{array}{l}75.3 \\
64.8 \\
55.0 \\
47.4 \\
61.5 \\
48.1 \\
50.2 \\
55.1 \\
43.4 \\
49.1 \\
45.7 \\
45.5 \\
49.8 \\
58.7 \\
70.2 \\
53.1 \\
61.3 \\
54.9 \\
49.8 \\
62.0 \\
51.3 \\
45.8 \\
49.8 \\
50.2 \\
61.2 \\
62.9 \\
36.2 \\
55.1 \\
51.9 \\
51.3 \\
59.5 \\
62.2\end{array}$ & $\begin{array}{r}82.5 \\
80.0 \\
82.0 \\
108.0 \\
81.0 \\
125.0 \\
97.5 \\
101.0 \\
120.5 \\
100.5 \\
99.6 \\
112.0 \\
86.4 \\
86.4 \\
86.0 \\
92.2 \\
91.5 \\
103.0 \\
94.4 \\
98.1 \\
115.0 \\
101.5 \\
105.0 \\
109.5 \\
104.0 \\
105.0 \\
85.5 \\
109.0 \\
114.0 \\
113.4 \\
102.5 \\
110.0 \\
114.0\end{array}$ & $\begin{array}{l}\mathbf{6 2 . 1} \\
51.9 \\
45.7 \\
51.3 \\
49.7 \\
60.3 \\
49.5 \\
55.9 \\
51.9 \\
49.4 \\
45.3 \\
50.7 \\
41.1 \\
50.6 \\
60.4 \\
49.5 \\
55.9 \\
56.3 \\
46.9 \\
50.6 \\
57.7 \\
48.3 \\
54.6 \\
52.4 \\
64.2 \\
53.7 \\
39.6 \\
62.3 \\
58.8 \\
52.5 \\
65.7 \\
70.9\end{array}$ \\
\hline \multirow{3}{*}{$\begin{array}{c}\text { Mean } \\
\text { Standard } \\
\text { deviation } \\
\text { Standard } \\
\text { error }\end{array}$} & 63.7 & 139 & 34.2 & 3,362 & 54.2 & 100.4 & 53.5 \\
\hline & 12.8 & 2.9 & 7.14 & 722 & 7.8 & 12.1 & 6.7 \\
\hline & 2.3 & 0.5 & 1.3 & 128 & 1.4 & 2.1 & 1.2 \\
\hline
\end{tabular}


TABLE VI

Exchangeable sodium and body water in patients with hepatic disease with edema and/or ascites

\begin{tabular}{|c|c|c|c|c|c|c|c|}
\hline Patient & $\begin{array}{l}\text { Weight } \\
\text { (Kilos) }\end{array}$ & $\underset{\substack{\text { Serum } \\
\text { sodium } \\
(m E q . / L .)}}{\cos }$ & $\begin{array}{l}\text { Anti- } \\
\text { pyrine } \\
\text { space } \\
\text { (Liters) }\end{array}$ & $\begin{array}{l}\text { Total } \\
\text { exchange- } \\
\text { able } \\
\text { sodium } \\
(m E q .)\end{array}$ & ${ }_{(\% \text { Body wt.) }}^{\frac{\text { A.S. }}{\mathrm{Wt}}}$ & $\frac{\text { TENa }}{(m E q . / L .)}$ & $\frac{\text { TENa }}{\text { Wt }}$ \\
\hline $\begin{array}{r}1 \\
2 \\
3 \\
4 \\
5 \\
6 \\
7 \\
8 \\
9 \\
10 \\
11 \\
12 \\
13 \\
14 \\
15 \\
16\end{array}$ & $\begin{array}{r}54.6 \\
\\
85.5 \\
86.2 \\
53.2 \\
55.0 \\
74.2 \\
68.2 \\
88.0 \\
111.0 \\
78.5 \\
48.0 \\
72.0 \\
56.0 \\
50.5\end{array}$ & $\begin{array}{l}128 \\
130 \\
126 \\
130 \\
140 \\
128 \\
136 \\
131 \\
130 \\
128 \\
134 \\
124 \\
125 \\
133 \\
130 \\
122\end{array}$ & $\begin{array}{l}36.5 \\
33.4 \\
37.8 \\
51.4 \\
53.0 \\
36.5 \\
37.2 \\
50.4 \\
43.4 \\
59.7 \\
46.0 \\
45.5 \\
27.0 \\
46.5 \\
37.0 \\
32.2\end{array}$ & $\begin{array}{l}2,830 \\
2,710 \\
3,400 \\
4,770 \\
6,120 \\
3,000 \\
3,130 \\
3,820 \\
3,620 \\
5,940 \\
4,320 \\
4,080 \\
2,070 \\
4,210 \\
3,380 \\
3,600\end{array}$ & $\begin{array}{l}65.9 \\
\\
60.1 \\
61.5 \\
68.6 \\
67.7 \\
67.9 \\
63.6 \\
67.8 \\
41.4 \\
58.0 \\
56.2 \\
64.6 \\
66.0 \\
63.8\end{array}$ & $\begin{array}{r}78.0 \\
81.4 \\
90.0 \\
96.0 \\
115.0 \\
82.5 \\
84.0 \\
76.0 \\
83.5 \\
98.0 \\
94.0 \\
89.0 \\
78.0 \\
90.0 \\
91.0 \\
117.0\end{array}$ & $\begin{array}{l}51.8 \\
\\
55.8 \\
71.0 \\
56.4 \\
57.0 \\
51.5 \\
53.0 \\
67.5 \\
39.3 \\
51.9 \\
43.2 \\
58.5 \\
60.4 \\
71.4\end{array}$ \\
\hline \multirow{3}{*}{$\begin{array}{l}\text { Mean } \\
\text { Standard } \\
\text { deviation } \\
\text { Standard } \\
\text { error }\end{array}$} & 70.0 & 130 & 43.0 & 3,810 & 61.0 & 90.3 & 56.2 \\
\hline & 14.5 & 4.6 & 8.82 & 1,097 & 7.15 & 11.6 & 9.3 \\
\hline & 4.0 & 1.18 & 2.3 & 284 & 1.98 & 3.0 & 2.6 \\
\hline
\end{tabular}

deuterium 47.6 liters, in the same edematous patients with heart disease (Table I). Hence, in patients with congestive heart failure and varying amounts of edema, antipyrine would appear to be a valid measure of body water.

Degree of equilibration of antipyrine between plasma and extravascular fuid

In most instances the concentration of antipyrine in plasma and in samples of large accumula- tions of extravascular fluid collected 10 to 24 hours after the injection of antipyrine was in good agreement (Table II).

\section{Exchangeable sodium in extravascular fluids}

On examining 13 of the sera and extravascular fluids for radioactivity, it was found that, at the end of 24 hours, each of the fluids had the same concentration of radioactivity as the simultaneously drawn serum.

TABLE VII

Exchangeable sodium and body water in patients with renal disease with edema

\begin{tabular}{|c|c|c|c|c|c|c|c|}
\hline Patient & $\begin{array}{l}\text { Weight } \\
\text { (Kilos) }\end{array}$ & $\underset{\substack{\text { Serum } \\
\text { sodium } \\
(m E q . / L .)}}{{ }^{\prime}}$ & $\begin{array}{l}\text { Anti- } \\
\text { pyrine } \\
\text { space } \\
\text { (Liters) }\end{array}$ & $\begin{array}{l}\text { Total } \\
\text { exchange- } \\
\text { able } \\
\text { sodium } \\
(m E q .)\end{array}$ & $\underset{(\% \text { Body wt.) }}{\frac{\text { A.S. }}{\mathrm{Wt}}}$ & $\frac{\text { TENa }}{(m E q . / L .)}$ & $\frac{\text { TENa }}{W_{\text {t }}}$ \\
\hline $\begin{array}{r}1 \\
2 \\
3 \\
4 \\
5 \\
6 \\
7 \\
8 \\
9 \\
10\end{array}$ & $\begin{array}{l}65.6 \\
82.3 \\
58.2 \\
73.7 \\
52.7 \\
82.4 \\
56.9 \\
60.4 \\
60.9 \\
65.0\end{array}$ & $\begin{array}{l}142 \\
139 \\
136 \\
137 \\
136 \\
137 \\
138 \\
134 \\
136 \\
132\end{array}$ & $\begin{array}{l}51.4 \\
62.2 \\
28.7 \\
49.0 \\
32.3 \\
52.8 \\
28.6 \\
44.2 \\
42.4 \\
40.0\end{array}$ & $\begin{array}{l}4,760 \\
5,660 \\
2,585 \\
4,390 \\
2,870 \\
3,960 \\
2,590 \\
3,860 \\
4,020 \\
3,590\end{array}$ & $\begin{array}{l}78.3 \\
75.5 \\
49.2 \\
66.5 \\
61.3 \\
64.1 \\
50.2 \\
73.2 \\
69.6 \\
61.4\end{array}$ & $\begin{array}{l}92.6 \\
91.0 \\
89.9 \\
89.6 \\
91.0 \\
75.0 \\
90.8 \\
87.5 \\
94.8 \\
90.0\end{array}$ & $\begin{array}{l}72.6 \\
68.7 \\
44.4 \\
59.5 \\
54.5 \\
48.1 \\
45.5 \\
64.0 \\
66.1 \\
55.2\end{array}$ \\
\hline \multirow{3}{*}{$\begin{array}{c}\text { Mean } \\
\text { Standard } \\
\text { deviation } \\
\text { Standard } \\
\text { error }\end{array}$} & 65.8 & 137 & 43.2 & 3,828 & 65.0 & 89.2 & 57.9 \\
\hline & 10.7 & 2.72 & 11.1 & 975 & 9.85 & 5.1 & 9.9 \\
\hline & 3.46 & 0.91 & 3.7 & 325 & 3.28 & 1.7 & 3.3 \\
\hline
\end{tabular}


TABLE VIII

Summary of mean values

\begin{tabular}{lccccc}
\hline \hline & Controls & $\begin{array}{c}\text { Edematous } \\
\text { cardiacs }\end{array}$ & $\begin{array}{c}\text { Non- } \\
\text { Edematous } \\
\text { cardiacs }\end{array}$ & $\begin{array}{c}\text { Hepatic } \\
\text { disease }\end{array}$ & $\begin{array}{c}\text { Renal } \\
\text { disease }\end{array}$ \\
\hline Weight (Kilos) & 66.8 & 68.2 & 63.7 & 70.0 & 65.8 \\
Antipyrine space (Liters) & 35.0 & 37.9 & 34.2 & 43.0 & 43.2 \\
A.S./Kilo (\% Body wt.) & 52.0 & 55.5 & 54.2 & 61.0 & 65.0 \\
Total exchangeable sodium (mEq.) & 2896 & 4036 & 3362 & 3810 & 3828 \\
TENa/A.S. (mEq./Liter) & $\mathbf{8 1 . 3}$ & $\mathbf{1 0 8 . 2}$ & $\mathbf{1 0 0 . 4}$ & 90.3 & 89.2 \\
TENa/Kg. (mEq./kilo) & 43.7 & 59.9 & 53.5 & 56.2 & 57.9 \\
Number of subjects & 27 & $50^{*}$ & $33^{*}$ & $10^{*}$ & 10 \\
\hline
\end{tabular}

* In the edematous cardiac, non-edematous cardiac, and hepatic groups there were 4, 1, and 2 patients, respectively, whose weights were not obtainable.

Antipyrine space and antipyrine space as per cent of body weight

The edematous patients with hepatic or renal disease, had a significantly larger mean value for body water and body water expressed as per cent of body weight than any of the other groups (Tables VIII, XI C). The patients with edema due to heart disease had a small increase above normal that was not statistically different from the control. The group of patients with heart disease who were previously edematous, had a value closer to normal.

Total exchangeable sodium antipyrine space (Tables III to VIII)

In an effort to determine body sodium concentration, total exchangeable sodium and antipyrine space were related to each other. In the controls the resultant ratio expressed as milliequivalents of sodium per liter of body water was 81.3 with a standard deviation of 6.2 and a standard error of 1.2 (Table III). In each group of edematous patients with edema due to heart, renal or liver disease and in patients with heart disease who were previously edematous, the ratios of exchangeable sodium to body water were significantly higher than the controls (Table XI A).

The following mean values for the ratios were obtained : in patients with edema due to heart disease 108.2 (Table IV), hepatic disease 90.3 (Table VI), renal disease 89.2 (Table VII), and in treated heart disease patients who had lost their edema 100.4 (Table V, Figures 1, 2, and 3). The values for each of the four abnormal groups were significantly different from each other except that the value obtained in patients with edema due to renal disease was not significantly different

TABLE IX

Comparison of exchangeable sodium and body water in patients with heart disease when edematous and later when non-edematous *

\begin{tabular}{|c|c|c|c|c|c|c|c|c|c|c|c|}
\hline \multirow[b]{2}{*}{$\begin{array}{c}\text { Edema- } \\
\text { tous } \\
\text { patient }\end{array}$} & \multirow[b]{2}{*}{$\begin{array}{l}\text { Weight } \\
\text { (Kilos) }\end{array}$} & \multirow[b]{2}{*}{$\begin{array}{l}\text { Anti- } \\
\text { pyrine } \\
\text { space } \\
\text { (Liters) }\end{array}$} & \multirow[b]{2}{*}{$\frac{\text { A.S. }}{(\% \underset{\text { Wod. }}{\text { Boty }}}$} & \multirow[b]{2}{*}{ 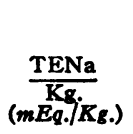 } & \multirow[b]{2}{*}{$\frac{\text { TENa }}{(m E \text { Q.S. } / L .)}$} & \multirow[b]{2}{*}{ Patient } & \multirow[b]{2}{*}{$\begin{array}{l}\text { Weight } \\
\text { (Kilos) }\end{array}$} & \multirow[b]{2}{*}{$\begin{array}{l}\text { Anti- } \\
\text { pyrine } \\
\text { space } \\
\text { (Liters) }\end{array}$} & \multirow[b]{2}{*}{$\frac{\text { A.S. }}{\text { Wt. }}$} & \multicolumn{2}{|c|}{ Non-Edematous } \\
\hline & & & & & & & & & & $\frac{\mathrm{TENa}}{\left.\mathrm{Kg}_{(m E q .} / K_{g .}\right)}$ & $\frac{\text { TENa }}{\left(m \bar{E} q_{.} / L .\right)}$ \\
\hline $\begin{array}{r}2 \\
5 \\
8 \\
19 \\
20 \\
26 \\
27 \\
33 \\
37 \\
38 \\
40 \\
44 \\
49\end{array}$ & $\begin{array}{r}91.0 \\
75.4 \\
66.8 \\
110.5 \\
66.0 \\
64.5 \\
68.8 \\
55.0 \\
85.0 \\
71.0 \\
69.5 \\
62.3 \\
62.9\end{array}$ & $\begin{array}{l}43.9 \\
49.6 \\
41.7 \\
62.1 \\
43.5 \\
30.5 \\
35.7 \\
31.6 \\
47.6 \\
50.6 \\
44.8 \\
34.8 \\
33.5\end{array}$ & $\begin{array}{l}48.3 \\
65.8 \\
62.4 \\
56.2 \\
66.0 \\
47.3 \\
51.9 \\
57.4 \\
56.0 \\
71.3 \\
50.0 \\
55.8 \\
53.3\end{array}$ & $\begin{array}{l}49.0 \\
63.7 \\
60.2 \\
63.8 \\
62.5 \\
63.4 \\
66.8 \\
72.8 \\
56.0 \\
71.9 \\
75.1 \\
66.9 \\
59.8\end{array}$ & $\begin{array}{r}101.0 \\
97.4 \\
96.0 \\
113.5 \\
140.0 \\
134.0 \\
128.5 \\
115.6 \\
100.0 \\
105.0 \\
116.5 \\
119.5 \\
112.0\end{array}$ & $\begin{array}{r}14 \\
15 \\
3 \\
16 \\
17 \\
23 \\
24 \\
26 \\
25 \\
32 \\
29 \\
30 \\
11\end{array}$ & $\begin{array}{l}70.0 \\
61.3 \\
66.8 \\
77.8 \\
41.7 \\
54.5 \\
61.3 \\
52.3 \\
74.1 \\
60.0 \\
57.7 \\
52.0 \\
61.8\end{array}$ & $\begin{array}{l}41.1 \\
43.0 \\
37.3 \\
41.3 \\
29.2 \\
25.0 \\
30.6 \\
32.0 \\
37.2 \\
35.8 \\
31.8 \\
27.0 \\
28.2\end{array}$ & $\begin{array}{l}\mathbf{5 8 . 7} \\
\mathbf{7 0 . 2} \\
\mathbf{5 5 . 9} \\
\mathbf{5 3 . 1} \\
\mathbf{6 1 . 3} \\
\mathbf{4 5 . 8} \\
\mathbf{4 9 . 8} \\
\mathbf{6 1 . 2} \\
\mathbf{5 0 . 2} \\
\mathbf{5 2 . 7} \\
\mathbf{5 5 . 1} \\
\mathbf{5 1 . 9} \\
\mathbf{4 5 . 7}\end{array}$ & $\begin{array}{l}50.6 \\
60.4 \\
45.7 \\
49.5 \\
55.9 \\
48.3 \\
54.6 \\
64.2 \\
52.4 \\
65.8 \\
62.3 \\
58.8 \\
45.3\end{array}$ & $\begin{array}{r}86.4 \\
86.0 \\
82.0 \\
92.2 \\
91.5 \\
105.0 \\
109.5 \\
105.0 \\
104.0 \\
110.0 \\
114.0 \\
113.0 \\
99.6\end{array}$ \\
\hline Mean & 72.2 & 42.3 & 57.0 & 64.0 & 113.7 & & 61.2 & 33.9 & 54.6 & 54.6 & 99.9 \\
\hline
\end{tabular}

* These are the same patients. The patient number corresponds to the numbers in Tables IV and V. 
TABLE $X$

Exchangeable sodium and body water in a patient with heart disease followed for five months

\begin{tabular}{|c|c|c|c|c|c|}
\hline Date & Edema & $\underset{\left(K_{\mathbf{g}}\right)}{\mathbf{W} t}$ & $\begin{array}{c}\text { Antipyrine } \\
\text { 8pace } \\
\text { (Liters) }\end{array}$ & $\begin{array}{c}\text { TENa } \\
(m E q .)\end{array}$ & $\begin{array}{l}\text { TENa/A.S. } \\
\left(m E_{Q .} / L \text { iter }\right)\end{array}$ \\
\hline $\begin{array}{c}11 / 18 \\
12 / 11 \\
1 / 5 \\
3 / 16 \\
4 / 28\end{array}$ & $\begin{array}{c}++ \\
0 \\
0 \\
0 \\
0\end{array}$ & $\begin{array}{l}68.6 \\
61.3 \\
61.4 \\
61.4 \\
65.0\end{array}$ & $\begin{array}{l}35.3 \\
32.3 \\
30.6 \\
32.2 \\
32.9\end{array}$ & $\begin{array}{l}4,590 \\
4,220 \\
3,350 \\
2,880 \\
2,600\end{array}$ & $\begin{array}{r}129 \\
130 \\
109 \\
89 \\
79\end{array}$ \\
\hline
\end{tabular}

than the one obtained in patients due to hepatic disease (Table XI A).

Of 33 non-edematous patients with heart disease, eight had ratios of total exchangeable sodium to antipyrine space which were in the normal range (Table $\mathrm{V}$ ). The average of the ratios for the entire group was high, 100.4 and significantly different from that of the other groups of patients and the control group (Table XI A). There was no relationship between the value for the $\mathrm{TENa/}$

TABLE XI

Statistical analyses

\begin{tabular}{|c|c|c|c|c|c|}
\hline & \multirow[b]{2}{*}{ Control } & \multirow{2}{*}{$\begin{array}{l}\text { Edema } \\
\text { due to } \\
\text { heart } \\
\text { disease }\end{array}$} & \multirow{2}{*}{$\begin{array}{l}\text { Previously } \\
\text { edematous } \\
\text { with heart } \\
\text { disease }\end{array}$} & \multicolumn{2}{|c|}{ Edema due to: } \\
\hline & & & & $\begin{array}{l}\text { Hepatic } \\
\text { disease }\end{array}$ & $\begin{array}{l}\text { Renal } \\
\text { disease }\end{array}$ \\
\hline \multicolumn{6}{|l|}{ A) TENa/A.S. p (Probability) } \\
\hline Control $(N=27)$ & & $>0.01$ & $>0.01$ & $>0.01$ & $>0.01$ \\
\hline \multicolumn{6}{|l|}{ Patients with edema due to: } \\
\hline $\begin{array}{l}\text { Heart disease }(\mathrm{N}=50) \\
\text { Hepatic disease }(\mathrm{N}=16) \\
\text { Renal disease }(\mathrm{N}=10) \\
\text { Heart disease previously edematous }(\mathrm{N}=33)\end{array}$ & $\begin{array}{l}>0.01 \\
>0.01 \\
>0.01 \\
>0.01\end{array}$ & $\begin{array}{l}>0.01 \\
>0.01 \\
>0.01\end{array}$ & $\begin{array}{l}>0.01 \\
>0.01 \\
>0.01\end{array}$ & $\begin{array}{r}>0.01 \\
0.50 \\
>0.01\end{array}$ & $\begin{array}{r}>0.01 \\
0.50 \\
>0.01\end{array}$ \\
\hline \multicolumn{6}{|l|}{ B) TENa/Kg. p (Probability) } \\
\hline Control & & $>0.01$ & $>0.01$ & $>0.01$ & $>0.01$ \\
\hline \multicolumn{6}{|l|}{ Patients with edema due to: } \\
\hline $\begin{array}{l}\text { Heart disease } \\
\text { Hepatic disease } \\
\text { Renal disease } \\
\text { Heart disease previously edematous }\end{array}$ & $\begin{array}{l}>0.01 \\
>0.01 \\
>0.01 \\
>0.01\end{array}$ & $\begin{array}{r}0.50 \\
0.50 \\
>0.01\end{array}$ & $\begin{array}{r}>0.01 \\
0.50 \\
0.50\end{array}$ & $\begin{array}{l}0.50 \\
0.50 \\
0.50\end{array}$ & $\begin{array}{l}0.50 \\
0.50 \\
0.50\end{array}$ \\
\hline \multicolumn{6}{|l|}{ C) A.S. \& A.S./Kg. p (Probability) } \\
\hline Control & & 0.50 & 0.50 & $>0.01$ & $>0.01$ \\
\hline \multicolumn{6}{|l|}{ Patients with edema due to: } \\
\hline $\begin{array}{l}\text { Heart disease } \\
\text { Hepatic disease } \\
\text { Renal disease } \\
\text { Heart disease previously edematous }\end{array}$ & $\begin{array}{r}0.50 \\
>0.01 \\
>0.01 \\
0.50\end{array}$ & $\begin{array}{r}>0.01 \\
>0.01 \\
0.50\end{array}$ & $\begin{array}{r}0.50 \\
>0.01 \\
>0.01\end{array}$ & $\begin{array}{r}>0.01 \\
0.50 \\
>0.01\end{array}$ & $\begin{array}{r}>0.01 \\
0.50 \\
>0.01\end{array}$ \\
\hline
\end{tabular}

D) Comparison of Heart Disease, Previously Edematous to Control

$\begin{array}{lccc} & \begin{array}{c}\text { Previously } \\ \text { edematous } \\ \text { with heart } \\ \text { disease } \\ (\mathrm{N}=33)\end{array} & \begin{array}{c}\text { Control } \\ (\mathrm{N}=27)\end{array} & \begin{array}{c}\text { p } \\ \text { Weight }\end{array} \\ \text { Antipyrine space } & 63.7 & 66.8 & 0.50 \\ \text { Total exchangeable Na } & 34.2 & 35.0 & 0.50 \\ \text { TENa/A.S. } & 3362 & 2896 & >0.01 \\ \text { TENa/Kg. } & 100 & 81 & >0.01 \\ \end{array}$

E) Patients with Edema due to Heart Disease Compared to Patients with Cirrhosis with Similar Amounts of Body Water as Per Cent of Body Weight

Patients with edema due to: Number $\quad \begin{gathered}\text { \%.S. as } \\ \text { weight }\end{gathered}$ TENa/Kg.

p

Heart disease

$14 \quad 61.7 \quad 65.2$

Hepatic disease

\begin{tabular}{lll}
14 & 62.4 & 56.2 \\
\hline
\end{tabular}


TABLE XII

Theoretical calculations

\begin{tabular}{|c|c|c|c|c|}
\hline & $\begin{array}{c}\text { Weight } \\
\left(K_{\left.K_{g}\right)}\right)\end{array}$ & $\begin{array}{c}\text { Body water } \\
\text { (Liters) }\end{array}$ & $\begin{array}{c}\text { \% Body } \\
\text { weight }\end{array}$ & TENa \\
\hline $\begin{array}{l}\text { Control: } \\
\quad \text { Serum } \mathrm{Na}=140 \mathrm{mEq} . / \mathrm{L} . \\
\text { (Assumed for all calculations) }\end{array}$ & 67.0 & $\begin{array}{l}35.0 \\
\text { TENa/A.S. }=82.5 \\
\text { TENa/Kg. }=43.7\end{array}$ & 53.0 & 2,896 \\
\hline $\begin{array}{l}\text { I. Loss of } 10 \mathrm{Kg} \text {. of weight in form of pro- } \\
\text { tein or } 7.5 \text { liters of intracellular water loss }\end{array}$ & 57.0 & $\begin{array}{l}27.5 \\
\text { TENa/A.S. }=105.0 \\
\text { TENa/Kg. }=50.9\end{array}$ & 48.2 & 2,896 \\
\hline II. A) Gain of 10 liters of extracellular water & 77.0 & $\begin{array}{l}45.0 \\
\text { TENa/A.S. }=95.2 \\
\text { TENa/Kg. }=55.8\end{array}$ & 58.5 & 4,296 \\
\hline B) Gain of 15 liters of extracellular water & 82.0 & $\begin{array}{l}50.0 \\
\text { TENa/A.S. }=100.0 \\
\text { TENa/Kg. }=61.0\end{array}$ & 61.0 & 4,996 \\
\hline III. Loss of $10 \mathrm{Kg}$. of weight in form of fat & 57.0 & $\begin{array}{l}33.0 \\
\text { TENa/A.S. }=88.0 \\
\text { TENa/Kg. }=51.0\end{array}$ & 58.0 & 2,896 \\
\hline $\begin{array}{l}\text { IV. Loss of } 10 \mathrm{Kg} \text {. of weight in form of pro- } \\
\text { tein or } 7.5 \text { liters of intracellular water plus } \\
\text { gain of } 10 \text { liters of extracellular fluid }\end{array}$ & 67.0 & $\begin{array}{l}37.5 \\
\text { TENa/A.S. }=114.0 \\
\text { TENa/Kg. }=64.0\end{array}$ & 56.0 & 4,296 \\
\hline
\end{tabular}

A.S. ratio obtained and the length of time a particular patient was edema free.

Body water measurements in this group of

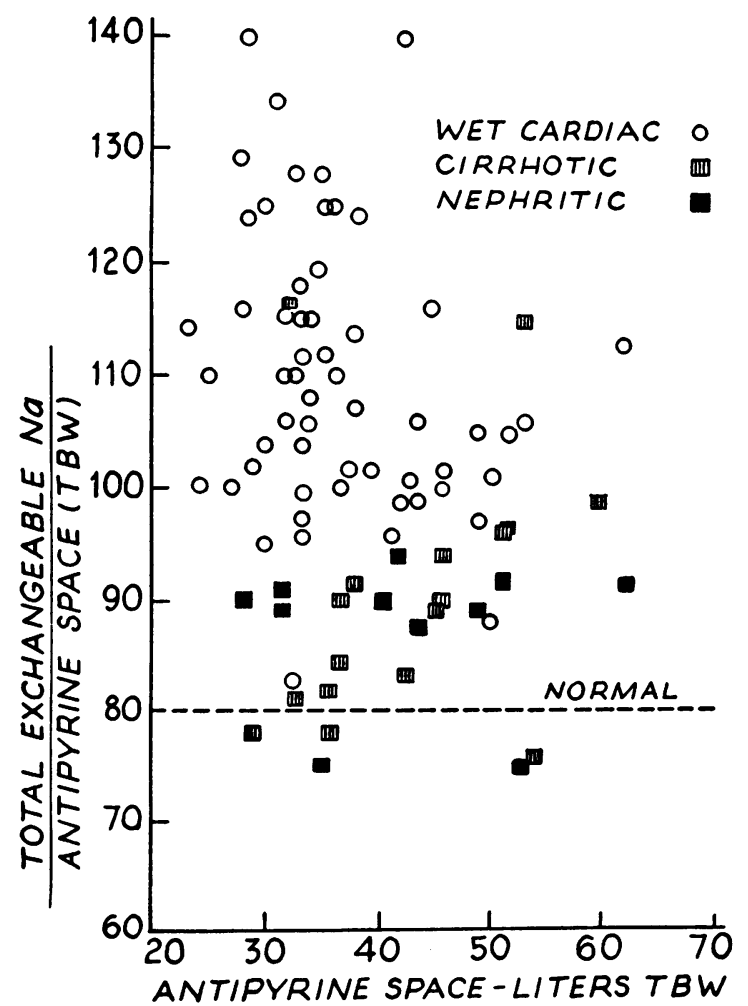

Fig. 1. TENa/A.S. IN Edematous Patients $\mathrm{TBW}=$ Total Body Water. non-edematous patients with heart disease were not significantly different from the normal values. Exchangeable sodium proved to be significantly greater (Table XI D).

Two patients with renal disease had a ratio of body sodium to body water that returned to the normal range after a diuresis and when they were free of edema.

These data, therefore, indicate that patients with edema due to heart disease had a higher mean ratio of sodium to water in their bodies than did patients with excess of fluid accumulation due to renal or liver disease. This high ratio of sodium to water persisted in the bodies of the majority of patients with heart disease after their edema was gone.

Exchangeable sodium and body water in patients with heart disease before and after diuresis (Table IX)

Total exchangeable sodium and water were studied in 13 patients with edema due to heart disease and again when there was no clinical edema and their weights were constant for at least three weeks. The average weight decreased $9.0 \mathrm{~kg}$., body water 8.4 liters, TENa per liter of body water $13.8 \mathrm{mEq}$., and TENa per kilogram body weight $9.4 \mathrm{mEq}$. The final values for TENa per liter body water and TENa per $\mathrm{kg}$. body weight were 


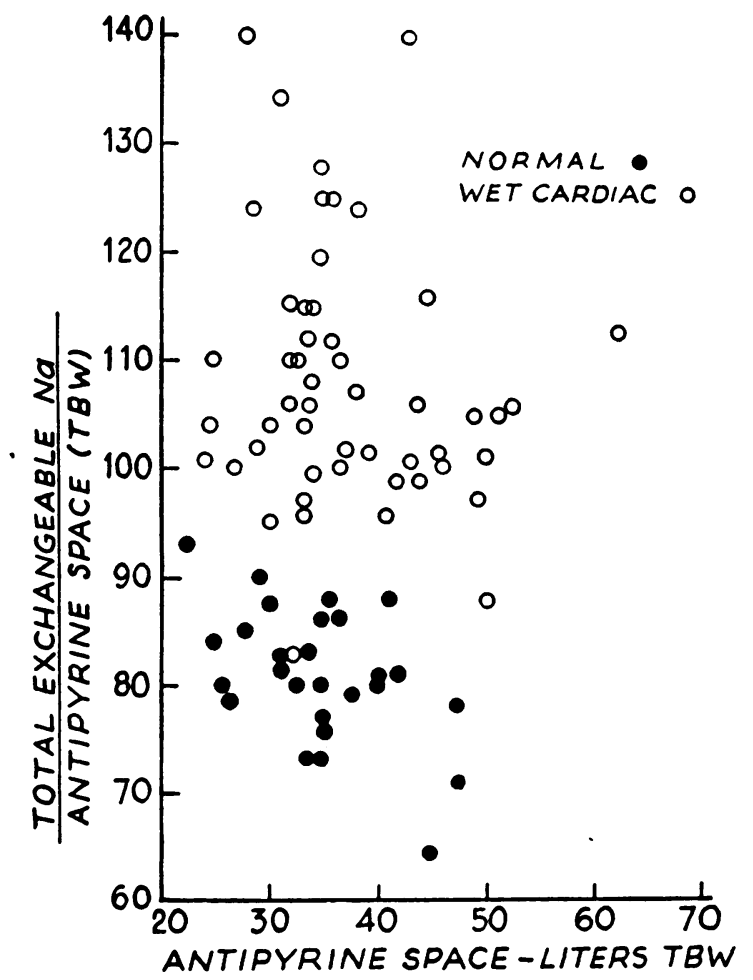

Fig. 2. TENa/A.S. IN Patients with Edema Due to Heart Disease and in Normals

TBW $=$ Total Body Water.

still significantly elevated as compared with the control. TENa per liter, 99.9 with control 81.3, TENa per kg. 54.6 with control 43.7. The mean figures for weight loss and water loss were in good agreement. However, the individual data showed a good deal of variation and no consistent cause could be established to explain the lack of correlation of the weight and water data in individual patients.

Serial determinations of body water and total exchangeable sodium

In one edematous patient with heart disease serial determinations were made over a 5-month period (Table $\mathrm{X}$ ). He lost his edema during the first three weeks of hospitalization. As was characteristic of other patients of this type, his TENa per liter of body water remained elevated, 130 $\mathrm{mEq}$. per liter. During the subsequent five months he did not lose weight or body water but his exchangeable sodium steadily decreased (1620 $\mathrm{mEq}$.) until the value reached the normal range.
Since his body water did not increase, the gain in weight during the last month of observation was considered to be due to a probable gain in adipose tissue. Thus, the patient continued to lose sodium after he became edema free. The clinical evaluation of his edema free state was substantiated by the observations of constant figures for body water during the four months of study.

Body weight and total exchangeable sodium. Total exchangeable sodium/body weight (kilos) (Table III to VIII).

To substantiate the finding of the possible abundance of sodium in patient with heart disease an analysis was made of total exchangeable sodium per kilogram body weight.

An analysis of exchangeable sodium expressed in $\mathrm{mEq}$. per $\mathrm{kg}$. body weight gave the following results (Tables III to VII) : control 43.7 ; edematous patients with heart disease 59.9 ; with hepatic disease 56.2 ; with renal disease 57.9 ; non-edematous patients with heart disease $53.5 \mathrm{mEq}$. per $\mathrm{kg}$. The values for each group of the edematous sub-

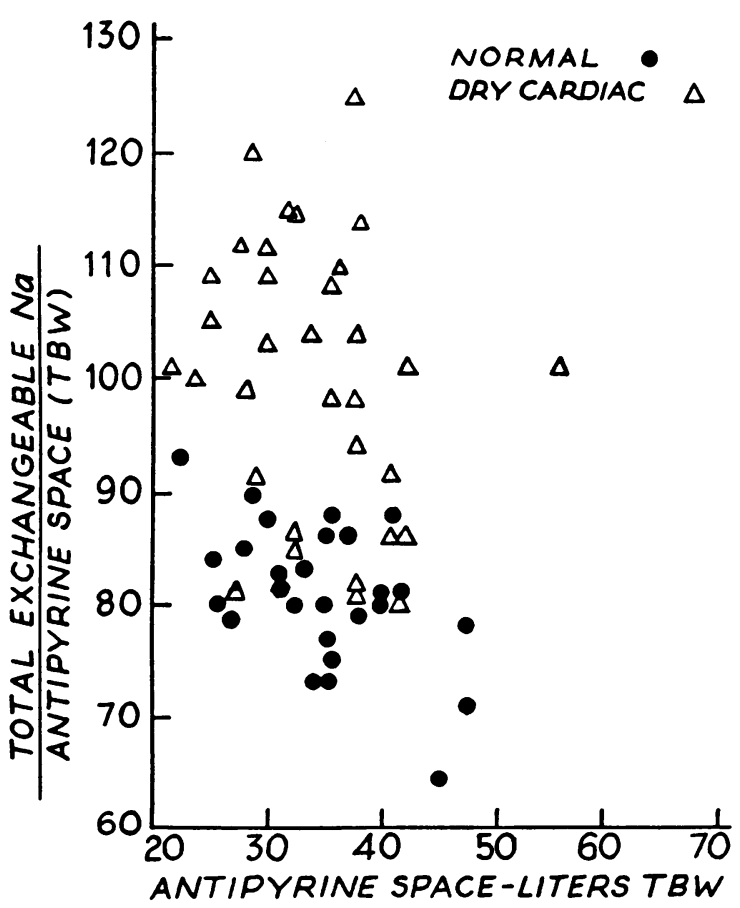

Fig. 3. TENa/A.S. In Patients with Heart Disease Who Were Previously Edematous aNd in NoRMaLS

TBW $=$ Total Body Water. 
jects differed significantly from the normal of $43.7 \mathrm{mEq}$. per kilo but not from each other (Table XI B).

Since body weight is the sum of various components which are variable from individual to individual, an attempt was made to compare individuals in the edematous group in whom body water composition, as per cent of body weight, was similar. For this purpose patients from the edematous group with heart disease were compared to patients with hepatic disease who had similar body water composition. When the values for sodium per kilo body weight of these chosen groups were then statistically analyzed, these groups were probably significantly different from each other. The $p$ value of this analysis was 0.02 (Table XI E).

The patients with heart disease who were previously edematous and at the time of the study were edema free had a high average value for sodium per kilo body weight. When compared to the controls, the value for sodium per kilo body weight obtained was significantly greater. When compared to the values for the edematous patients with heart disease the ratio was lower (Table XI B). Body weight of this group of non-edematous patients with heart disease was not significantly different from the normal whereas exchangeable sodium was higher.

\section{DISCUSSION}

The data of the edematous groups of patients indicate that patients with edema due to heart disease differed in several ways from the controls and the patients with edema due to hepatic or renal disease. 1) The ratios of exchangeable sodium to body water were greater in the patients with heart disease than in the controls and in the other edematous groups of patients. 2) The values for body water and body water as per cent body weight were not different in the edematous patients with heart disease when compared to the controls. The figures for body water and body water as per cent of body weight in the patients with edema due to hepatic or renal disease were greater than the controls and the values obtained in patients with edema due to heart disease.

The data of the group of patients with heart disease who were previously edematous and at the time of study clinically free of edema indicate that the average of the ratios of body sodium to body water was greater than the control group. The average value for body water and body water as per cent of body weight was very close to normal.

In considering the possible interpretations of the data obtained for the ratios of body sodium to body water in the edematous patients with heart disease, the ratios and their relationship to body composition bear consideration. Thus, the increase in the average of the ratios of body sodium to body water in edematous patients with heart disease as compared to patients with edema due to other causes may be due either to an abundance of sodium as related to water above that expected to be due to extracellular fluid accumulation, or a change in body composition which would alter this ratio without the existence of an excess of sodium.

The concentration of sodium is different in the various compartments of the body and changes in the ratio of body sodium to body water can result from increases or decreases in any of the fluid compartments. The direction and the magnitude of change will depend upon the sodium concentration of the fluid gained or lost. These theoretical results are calculated in the accompanying list of Theoretical Calculations I-IV, Table XII. The calculations involving loss of body protein or fat assume values for water of these components derived by other investigators $(7,8)$.

Clinical edema is the result of the accumulation of an abundance of extracellular fluid. Since sodium exists in a concentration of approximately $140 \mathrm{mEq}$. per liter in extracellular fluid and in a lower concentration in cellular fluids, the accumulation of edema leads to a proportionately greater increase in total exchangeable sodium than in total body water. Theoretical Calculation II A shows this for a gain, as edema fluid, of 10 liters of extracellular water. An increase will result in the value for sodium per liter of body water. The estimated accumulation of edema in most of the patients with heart disease in this study was 10 to 12 liters. However, the individual values and the average of the ratios obtained in edematous patients with heart disease were much higher than the expected increases (averages: theoretical 95 $\mathrm{mEq}$. per liter, $55.8 \mathrm{mEq}$. per kg.; observed 108 $\mathrm{mEq}$. per liter, $60 \mathrm{mEq}$. per kg.). The patients with massive edema due to cardiac disease had 
ratios far above 108. The high average of the ratios in the heart disease group could, therefore, be due to an increase of sodium above that anticipated as extracellular fluid edema. The renal and hepatic disease groups, exhibited the theoretical change expected in individuals with straightforward extracellular fluid accumulation of moderate severity. Values of this order of magnitude for patients with cirrhosis of the liver have been reported by others (9).

Loss of body weight may also affect sodium and water relationships. This would not occur if the loss were in the form of fat (Calculation III). However, if the loss was in the form of cellular protein and, assuming this to be 75 per cent sodium free water (8), this loss of intracellular water would produce a theoretical increase in body sodium to body water ratio (Calculation I). This value would increase still further if extracellular edema fluid were added to the individual with loss of cellular mass (Calculation IV).

The data obtained for body water indicate that patients with congestive heart failure probably lose intracellular water and gain extracellular water during the course of their illness. Individuals who just accumulate edema fluid should have an increase in body water but body water (expressed either as liters or as per cent body weight) did not increase in the edematous patients with heart disease. These findings support the probability that some loss of intracellular or other fluid almost balanced the simultaneous gain of extracellular water. Therefore, the observed high ratio of body sodium to water may be due to loss of cellular water and edema fluid accumulation, or to a combination of loss of cellular water, extracellular fluid accumulation and an additional excess of sodium.

In substantiating the occurrence of the combination of events in the patients with congestive heart failure, the data of the non-edematous group of patients with heart disease, indicating that they had an absolute abundance of sodium, are of considerable value. Several observations substantiate the conclusion concerning the existence of an excess sodium in these non-edematous patients.

1) The statistical analysis of the data of nonedematous heart disease group, as compared to the controls, showed that they differed only in that total exchangeable sodium was significantly greater
(Table XI C). Body weight and body water were in the normal range. In the group of thirteen patients followed from the edematous to the nonedematous state a loss of water and sodium occurred, but an abnormally high ratio of body sodium to water persisted as a consequence of high total exchangeable sodium (Table IV). The increment in the average total exchangeable sodium in the whole group of non-edematous heart disease patients can be accounted for by a theoretical increase over the normal of four liters of extracellular fluid supplying an abundance of $560 \mathrm{mEq}$. of sodium. The presence of this amount of edema fluid could escape clinical detection. It may, therefore, be proposed that the high exchangeable sodium may be due to an increase of extracellular fluid that could not be observed on clinical examination. However, the value of 4 liters or 560 $\mathrm{mEq}$. of sodium is the mean for the group and includes data of 8 subjects who fall in the normal range and the data of 12 subjects whose fluid accumulation on the basis of the same type of calculation would be between 5 and 10.4 liters. The latter orders of magnitude of extracellular fluid accumulation would have been evident as edema.

2) Since the body water data imply a loss of cellular water in patients with congestive heart failure, it would be anticipated that after edema is gone, patients with heart disease would be comparable to individuals who have lost cellular water as a result of other types of disease. Included in the group of controls were five patients with advanced malignancies. If loss of weight in neoplastic diseases is a result of cellular loss, the anticipated theoretical increase in the ratio of body sodium to water would be the result of a decrease in body water with no change in total exchangeable sodium (Calculation I). Only a small increase in the average ratio was observed (Table III) in the patients with malignant disease (87 $\mathrm{mEq}$. per L., body water). This was due to a decrease in body water and exchangeable sodium. Apparently these individuals lost extracellular water as well as cellular water.

The data of the heart disease patients with no edema differ from the data obtained in the patients with malignant diseases. The non-edematous heart disease patients considered as a group as well as the thirteen patients followed from the edematous to the non-edematous phase of their 
disease had no decrease in body water when compared to the controls but exchangeable sodium was increased. These observations indicate that intracellular water that was lost in the congestive phase of heart failure was replaced, and some retention of non-extracellular fluid sodium was still present.

3) The study on patient McL. (Table X) indicated that this patient with heart disease had a continued decrease in exchangeable sodium from a high to a normal level over a period of five months after edema had clinically disappeared and there was objectively no change in body weight and body water. This patient, therefore, had an excess of sodium and not of water when he reached the edema free status. Subsequently he lost his excess of sodium without changing his body water.

These data, therefore, suggest that the nonedematous patient with heart disease has an abundance of sodium and it would be reasonable to conclude that the high ratio of body sodium to water in the edematous state due to heart disease is not entirely the result of a loss of cellular water and simultaneous accumulation of extracellular fluid but is in part due to the existence of an absolute abundance of sodium over water.

The data obtained in the patients with hepatic or renal disease may be due to simple extracellular fluid accumulation or to a combination of extracellular and intracellular fluid accumulation and an excess of sodium. An increase in intracellular sodium poor fluid could decrease an otherwise high ratio of body sodium to water. This possibility cannot be ruled out with the present data. The observations that two patients with renal disease had normal ratios when they recovered would suggest that extracellular fluid accumulation alone was present in their edematous state.

The conclusion concerning an excess of sodium in patients with heart disease would receive further support if the total sodium to body weight ratio in these individuals was as significantly elevated as the total sodium to body water ratio. The ratio of body sodium to body weight of the non-edematous patients with heart disease proved to be statistically different from the other groups of patients.

In the edematous patients with heart disease the total sodium to body weight ratio was not statisti- cally different from the ratios of the other edematous groups. The use of body weight as a standard of reference in this type of analysis is not ideal. Body weight is the sum of weights of different tissues and fluids of the body and variations in these components (e.g., fat) may occur which are not related to water or sodium. The edematous groups of patients in this study were heterogeneous in their body weights and not enough patients with hepatic or renal disease were studied to make this heterogeneity comparable in all the three edematous states. For this reason, patients from hepatic and heart disease groups who had similar amounts of body water as a per cent of body weight were separately compared to each other in regard to their sodium to body weight ratio. The results of this comparison (Table XI D) indicated that the difference between these two groups of edematous patients was probably significant.

Some questions may be raised concerning the methods used in investigating these groups of patients. Validation of the antipyrine space as a measure of body water in patients with edema due to heart disease, is provided by the deuterium dilution data and extravascular fluid equilibrium data. It would be expected that the accumulation of edema would lead to an increase in water as a per cent of body weight. This was true of the edematous patients with liver or renal disease. The edematous patients with heart disease, however, had only a slight increase. The latter observation has been made by others using tritium as a measure of body water (3).

Twenty-four-hour exchangeable sodium is probably a measure of most of the sodium in the body. All the sodium in bone does not equilibrate in twenty-four hours with radiosodium $(10,11)$. However, this unequilibrated portion of sodium may not be important in the physiological sodium pool of the body. To explain the data presented on the basis of some artefact in the measurement of exchangeable sodium, it would have to be postulated that in the patient with heart disease there is a pool of sodium which becomes exchangeable, and is not exchangeable in the normal or the patient with renal or hepatic disease. This possibility seems remote but cannot be ruled out.

The foregoing data and considerations indicate that patients with heart disease who have edema 
retain more sodium than water in their bodies as compared to patients with edema due to liver or renal disease. Patients with heart disease in contrast to patients with edema due to hepatic or renal disease probably lose intracellular water as they gain extracellular water. Individuals with heart disease store excess sodium for a variable period of time after they are edema free. An explanation for the defect concerning sodium distribution in patients with heart disease may be sought amongst the following: 1) These individuals have exchanged extracellular sodium for some other intracellular ion and have thereby retained sodium in excess of their total body water. 2) Excess sodium has been stored in some depot such as bone, cartilage or connective tissue. 3) Sodium may be bound to an extracellular (12) or intracellular tissue constituent, a situation in which it would be exchangeable but would not exert the anticipated osmotic effect for sodium in these biological fluids.

\section{SUM MARY}

1. Body water and total exchangeable sodium have been determined in normals, patients with edema due to heart, renal or hepatic disease and in patients with heart disease who were previously edematous.

2. Body water was determined with antipyrine. The results obtained with antipyrine were compared to body water determined with deuterium in nine edematous patients with congestive heart failure and were found to be in very close agreement.

3. Edematous patients with heart disease have less body water and body water as a per cent of body weight than do patients with edema due to renal or hepatic disease indicating a probable loss of intracellular along with a simultaneous gain of extracellular water.

4. Edematous patients with heart disease have a higher ratio of body sodium to body water in their bodies than do edematous patients with hepatic or renal disease which is considered to result from a loss of intracellular water, gain of extracellular water, and an absolute increase in body sodium. This conclusion is supported by the ob- servation that an excess of body sodium over body water persists in patients with heart disease who have been rendered edema free.

5. The data imply the existence of different metabolic defects concerning sodium and water distribution in these clinical conditions associated with edema formation.

\section{REFERENCES}

1. Soberman, R., Brodie, B. B., Levy, B. B., Axelrod, J., Hollander, V., and Steele, J. M., The use of antipyrine in the measurement of total body water in man. J. Biol. Chem., 1949, 179, 31.

2. Brodie, B. B., Axelrod, J., Soberman, R. J., and Levy, B. B., The estimation of antipyrine in biological materials. J. Biol. Chem., 1949, 179, 25.

3. Prentice, T. C., Siri, W., Berlin, N. I., Hyde, G. M., Parsons, R. J., Joiner, E. E., and Lawrence, J. H., Studies of total body water with tritium. J. Clin. Invest., 1952, 31, 412.

4. Warner, G. F., Dobson, E. L., Rodgers, C. E., Johnston, M. E., and Pace, N., The measurement of total "sodium space" and total body sodium in normal individuals and in patients with cardiac edema. Circulation, 1952, 5, 915.

5. Forbes, G. B., and Perley, A., Estimation of total body sodium by isotopic dilution. I. Studies on young adults. J. Clin. Invest., 1951, 30, 558.

6. Miller, H., and Wilson, G. M., The measurement of exchangeable sodium in man using the isotope $24 \mathrm{Na}$. Clin. Sc., 1953, 12, 97.

7. Pace, N., and Rathbun, E. N., Studies on body composition. III. The body water and chemically combined nitrogen content in relation to that content. J. Biol. Chem., 1945, 158, 685.

8. Behnke, A. R., Jr., Physiologic studies pertaining to deep sea diving and aviation, especially in relation to the fat content and composition of the human body. Harvey Lect., 1941/2, 37, 198.

9. Gilder, H., Redo, S. F., Barr, D., and Child, C. G., III., Water distribution in normal subjects and in patients with Laënnec's cirrhosis. J. Clin. Invest., 1954, 33, 555.

10. Davies, R. E., Kornberg, H. L., and Wilson, G. M., Non-exchangeable sodium in the body. Biochem. et Biophys. acta, 1952, 9, 703.

11. Edelman, I. S., James, A. H., Baden, H., and Moore, F. D., Electrolyte composition of bone and the penetration of radiosodium and deuterium oxide into dog and human bone. J. Clin. Invest., 1954, $32,122$.

12. Manery, J. F., Water and electrolyte metabolism. Physiol. Rev., 1954, 34, 334. 